\title{
Música y Arquitectura
}

\author{
Music and Architecture
}

Carmen Garfias Santiago ${ }^{1}$

\section{RESUMEN}

Es de interés, siempre, la interacción entre disciplinas diferentes de la autora del presente artículo, ya sea por los estudios musicales como por la formación arquitectónica, de aquí la fuerte curiosidad en buscar conexiones entre estas dos disciplinas.

En este artículo, se trata de explicar las analogías, las relaciones, la complementariedad, los estímulos que han dado una a la otra y además la complicidad compositiva, a través de un recorrido que observa momentos significativos de la historia donde estas dos artes se acercan.

\section{Palabras clave}

Música y Arquitectura, Pitágoras y las relaciones armónicas, el monocorde y consonancias armónicas en el Renacimiento, Palladio y las relaciones musicales, Xenakis y Le Corbusier, fachada de la catedral de Ronchamp, Luigi Nono y Renzo Piano

\section{ABSTRACT}

The interaction between different disciplines of the author of this article is always of interest, either by the musical studies as by the formation of architecture, it is for the strong curiosity in search for connections between these two disciplines.

In this article, it comes to explaining the analogies, the relationships, the complementarity, the stimuli that have given one to other one and also the compositive complicity, across a trip that observes significant moments of the history where these two arts approach.

\section{Keywords}

Music and Architecture, Pythagoras and the harmonious relations, the monotone and consonances in harmony with the Renaissance, Palladio and the musical relationships, Xenakis and Le Corbusier, facade of the cathedral of Ronchamp, Luigi Nono and Renzo Piano

\section{INTRODUCCIÓN}

Si se habla de armonía, ritmo, composición, equilibrio, base, módulo, orden, proporción, simetría y unidad de medida, ¿̇se refiere a la música o a la arquitectura? Son expresiones comunes a estas dos formas de expresión humana; si se analizan algunos de estos significados, se observa que el concepto es común a las dos.

Por ejemplo, el término 'ritmo', la definición dice que es el movimiento marcado por la sucesión regular de elementos débiles y fuertes, o bien de condiciones opuestas o diferentes, es decir, un flujo de movimiento, controlado o medido, sonoro o visual, generalmente producido por un orden de elementos diferentes del medio en cuestión. Esta consideración se puede aplicar sea a la música como a la arquitectura.

Se examina la palabra 'armonía' se ve que sucede algo parecido. Una definición dice que armonía es el equilibrio de las proporciones entre las distintas partes de un todo, y su resultado siempre connota belleza. En música, el estudio de la armonía implica los acordes y su construcción, así como las progresiones

Arquitecta por la URP y por la Universitd degli Studi di Roma 1 La Sapienza. Estudios de piano y dirección Coral en el Conservatorio Nacional de Música. Especialista en Restauro del monumento. 
de acordes y los principios de conexión que los rigen. La armonía como práctica musical es la transformación en un conjunto de reglas de supuesta validez, que permite encadenar notas superpuestas, este estudio puede tener base científica, pero el resultado es muy amplio y personal.

En arquitectura, armonía es la disposición o distribución ordenada y congruente de los elementos que componen una obra artística.

Si se considera el proceso creativo, el arquitecto que proyecta una obra y el compositor que concibe una música utilizan secuencias, disponen energías en el tiempo que ponen a disposición y a beneficio de quien lo disfruta. La música se escucha durante tiempo, y la arquitectura se goza en el espacio, las dos al servicio de la psique y del cuerpo. Son sorprendentes las afinidades, tanto que se pueden considerar el trabajo del compositor y el del constructor, como el de una arquitectura basada en el tiempo. Uno comunica arquitecturas en el tiempo, el otro comunica arquitecturas en el espacio. Uno en el espacio acústico, el otro en el espacio visivo, podríamos leerlo como componentes diferentes de una misma mirada de interés por el conocimiento. Es la unión entre arquitectura y música que se ha ido integrando durante la historia, ambas han tenido que encontrar nuevas formas de comunicación, ya sea involucrando los ámbitos sensoriales de la persona o interesando sobre todo las facultades auditivas. Son interesantes, a este respecto, las teorías de Bergson sobre el movimiento y la melodía, él dice: "Es con la audición que podemos concebir fácilmente el cambio sin cambiar nada: al escuchar una melodía tenemos, de hecho, la percepción clara de movimiento, sin que nada se mueva, o cambie. Para que haya movimiento, no debe haber necesariamente algo moviéndose ...". En esta idea se lee claramente el aspecto filosófico que une arquitectura y música, la relación que hay entre ellas ha conocido facetas diferentes en el transcurso de la historia.

En algunos momentos de la historia, la relación ha sido más mecánica o de respeto de proporciones matemáticas o numéricomusicales, teniendo un desarrollo análogo la una con la otra, se está hablando del período de la Antigua Grecia, de la Antigua Roma, y de cómo en el Renacimiento se retoman estos conceptos, adoptándolos como una verdadera ciencia. Posteriormente, el desarrollo de géneros musicales creó la necesidad de modificar la tipología arquitectónica en la que se representaban las obras, se cambió de las cortes o iglesias a teatros para la ejecución de obras teatrales o musicales. Mientras en el siglo XIX, en Europa, comienza un proceso de complicidad compositiva entre música y arquitectura que encuentra su mayor logro en el siglo XX, se puede citar el Pabellón de la Philips de Iannis Xenakis y Le Corbusier, donde la analogía ha sido de tipo simbólicofilosófico y la complicidad entre ellas compositivo-acústica; o teatros de Renzo Piano y Zaha Hadid. Mientras aún, de Le Corbusier, la fachada de la capilla de Notre Dame du Haut en Ronchamp donde la música ha sido inspiradora del proyecto de la fachada.

\section{Algunas relaciones entre música y arquitectura en las civilizaciones del pasado}

No se puede dar una fecha exacta en la que se pueda decir que esta relación tiene lugar, faltan fuentes documentarias, pero se sabe que los egipcios conocieron la música; en algunas representaciones rupestres -del período del Imperio Antiguo- o en bajorrelieves de tumbas se han reproducido instrumentos, arpas en lo específico, procedentes del arco de la caza al que se le había agregado una caja de resonancia en un extremo. Durante el reinado de Ramsés III, la ejecución del arpa, que había crecido como dimensión, estaba destinada a los sacerdotes. No hay fuentes escritas de partituras musicales o de la teoría musical que sabe utilizaron, pero por la posición de las manos se deduce que conocían los intervalos de octava, quinta y cuarta, no se puede decir que conocieron la armonía como se entiende actualmente, pero el uso de esos tonos demuestra que conocían las leyes que rigen la armonía musical. Se sabe también que a la arquitectura y a la escultura no se les atribuyó ningún valor de originalidad; la arquitectura para los egipcios tenía una función más simbólica que práctica. Podríamos decir que el nexo de unión entre 
música y arquitectura está en que las dos eran consideradas divinas.

De igual manera que en la cultura egipcia, en la cultura andina, no hay fuentes escritas, pero sí ceramios que hablan por sí solos, citado en el libro de Carlos Milla Villena, en el capítulo que trata sobre Arqueoastronomía, cuando se refiere a los espejos astronómicos, los ceramios Nazca donde hay 7 personas realizando una observación estelar a ritmo de tambor: tres astrónomos, dos ayudantes, un maestro que dirige el acto y un músico que acompaña el ritual, la misma escena se repite en un ceramio Maya de Veracruz donde hay 5 observadores acompañados por el ritmo musical. La técnica de observación astronómica se realiza desde el período precerámico (anterior a 1200 a.C.).

Es del mismo período la arquitectura monumental, principalmente de uso ceremonial. Se aprecia en los centros de Kotosh (2500 a.C.), primer ejemplo de arquitectura religiosa; La Galgada (2900 - 1800 a. C.), El Paraíso (2200 - 1900 a.C.) del período precerámico. Es fácil imaginar que en estos centros los rituales estuvieron acompañados por música. El nexo entre arquitectura y música sería que ambas estaban relacionadas con aspecto ritual y ceremonial.

\section{En la Antigua Grecia y en Roma.}

Algunas relaciones armónicas eran ya conocidas en el pensamiento griego, es Pitágoras (Samos aprox. 570 a.C. - Metaponto 495 a.C.) quien encuentra las relaciones en las consonancias musicales, digamos que define una tipología espacial entre los intervalos musicales, la distancia correspondiente entre dos notas concordantes (en consonancia) o en contraste (en disonancia) encerrando el concepto en una medida matemática fija. Así como el geómetra usa el compás o el físico la balanza, Pitágoras busca un instrumento para medir los intervalos. Se dice que, caminando por la calle, meditabundo, absorto en la idea de una ciencia musical, cuando escucha el sonido de unos martillos que llegan del taller de un herrero, él reconoce las consonancias fundamentales de cuarta, quinta y octava, entre la cuarta y la quinta la distancia de un tono. Pasa del plano perceptivo al plano numérico. ¿Cómo cuantificarlo? Entra al taller y mide el peso de los martillos, pasa seguidamente al terreno de la teoría y trata de entender el fenómeno. De regreso a su casa cuelga cuatro cuerdas iguales con pesos diferentes que estén en relación numérica de igualdad a $2 / 1$, $3 / 2$ y $4 / 3$. Individuando el peso justo calcula proporciones fijas, con el variar de los pesos, manteniendo constante la tensión obtenida colgando las cuerdas, encuentra relaciones matemáticas que definen la medida de la consonancia, midiendo el largo de la cuerda. Si el peso es el doble del largo, la cuerda tendida emite un sonido de octava, para la relación 3/2 emite la quinta y para 4/3 emite la cuarta. Este ejemplo lo realizó sucesivamente con el monocorde. Además, demostró que el sonido de octava contenía también los sonidos de quinta, de cuarta y otra sucesión de sonidos que se llama serie armónica. El concepto de Pitágoras es aún más amplio, está relacionado con la difusión filosófica del pitagorismo, donde conjuga una práctica política, poco respetuosa de valores tradicionales del mundo griego, con un continuo análisis de estructuras matemáticas describibles en la realidad. En sustancia, él no estaba buscando consonancias musicales, como filósofo, matemático, hombre de ciencia o político, sino que estaba interesado, metafóricamente, en la danza de los planetas que producen una música imperceptible para todos, menos para él. Esa música regida por proporciones matemáticas que, conectando el movimiento entre sí, armónico y ordenado de los planetas, armonizan dos elementos en oposición como forma y materia. Estaba interesado en otro aspecto de las consonancias, la música se vuelve un modelo para el análisis del número y de la realidad.

Se atribuye a Pitágoras y a sus discípulos el hecho de que, tal vez atraídos por las expresiones artísticas, hayan tratado de expresar en un número, en una expresión matemática la proporción perfecta, equivalente al número áureo 0,618 y al rectángulo áureo, o sea el rectángulo en que los lados están en tal proporción. Muchos estudiosos de matemáticas y de estética confirman que es un rectángulo con 
proporciones equilibradas desde el punto de vista matemático $y$ particularmente agradable a la vista del observador.

Podemos decir que la armonía es el punto de apoyo del pensamiento filosófico de Pitágoras, entendido en el sentido aritmético, musical y ético; y el número, la base de la armonía.

En Arquitectura, si se analiza la Acrópolis, la mayor parte de los templos fueron reconstruidos bajo la guía de Pericles durante la edad de oro de Atenas (460 - 430 a. C.), se vuelve a proponer el concepto arquitectónico de intervalo; se puede ver cómo los edificios han sido dimensionados según precisas reglas armónicas pitagóricas. En el Partenón (figura 1) se subraya una estructura rítmica entre las columnas y otros elementos (métopas y triglifos). Como en la fachada (figura 2), si se inscribe en un rectángulo donde un lado corresponde a la base y el otro lado a la altura máxima del tímpano, se tiene una relación áurea $(1: 1,618)$

Son también de este período, las armonías lidia, frigia y dórica, conocidas también como modos griegos; eran comportamientos melódicos construidos a partir de intervalos descompuestos y su escala asociada. Es importante comprender que lo que caracteriza y da el nombre a una escala es la sucesión precisa de intervalos, es decir, de tonos y semitonos que la componen. Según la tradición, los modos o armonías eran Dórico, Locrio, Frigio, Hipofrigio, Lidio, Hipolidio y Mixolidio. Los modos no son más que una organización de sonidos donde se establecen distancias entre tonos y semitonos entre los siete sonidos que la componen.

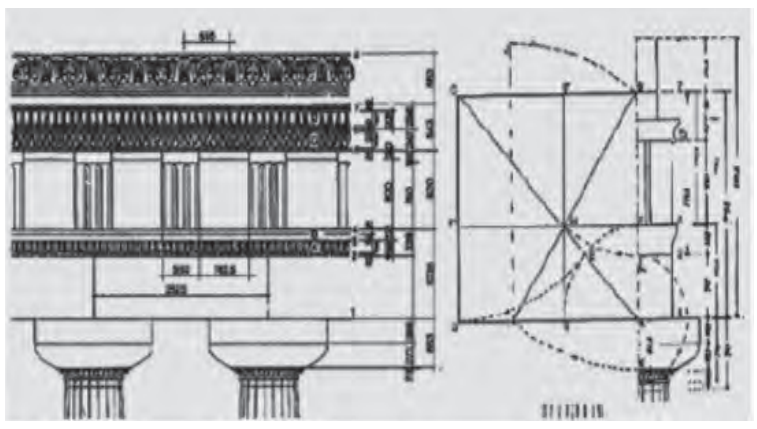

Figura 1. El Partenón, relaciones entre métopas, triglifos y otros elementos de la trabeación.

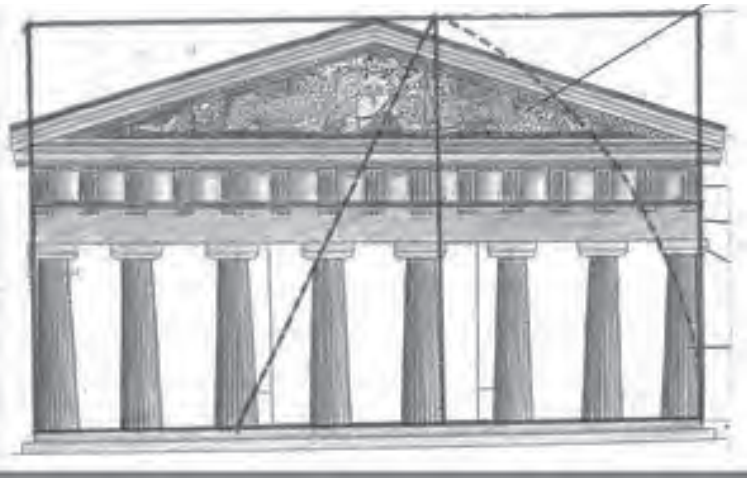

Figura 2. Rectángulo áureo que inscribe la fachada.

Los órdenes arquitectónicos son una gran novedad que los griegos introducen en el arte del construir. Consisten en una serie de reglas geométricas y matemáticas mediante las cuales cada elemento de un edificio está constantemente puesto en relación con las dimensiones del edificio en su conjunto. Aquí, también se habla de relaciones proporcionales, de consonancias armónicas. Está de más decir que los órdenes eran el Dórico, el Jónico y el Corintio cada uno con sus propias relaciones y características. Es clara la fusión entre música y arquitectura.

En la Antigua Roma, se tuvo una relación problemática con el arte; los romanos estaban interesados en cuestiones concretas y no en las cuestiones abstractas, fueron los más avanzados en cuanto a tecnología de su tiempo se refiere, la aplicaron en el campo de la ingeniería civil, materiales de construcción, hidráulica y transporte, su índole dura y sobria definida en siglos de guerras no les permitía pasar horas en tertulias tratando temas filosóficos o artísticos, digamos superficialmente y genéricamente que importaron la escuela griega, la de las proporciones matemáticas y numérico-musicales. Como dice Vitruvio (80 a.C. aprox - 15 a.C. aprox) en su obra Los diez libros de Arquitectura, Libro III, cap. I: " $\mathrm{La}$ composición de los templos es el resultado de la "simetría" y los arquitectos deben observar escrupulosamente los principios de la misma. Ésta surge de la proporción, que en griego se llama analoghía. La proporción es la conmensurabilidad sobre la base de una unidad determinada por sus miembros en cada estructura y en toda la obra, con la que el criterio de las relaciones modulares 
se traduce en práctica. $Y$, de hecho, ningún templo puede tener un principio racional de composición sin "simetría" ni proporción, si no se ha adherido al principio racional precisamente definido por los miembros de un hombre de formas proporcionadas.".

\section{Relaciones armónicas en el renacimiento}

No se puede hablar de relaciones armónicas o consonancias musicales sin nominar a los líderes y teóricos Alberti, Serlio y Palladio. Dos obras de Alberti son importantísimas: De re aedificatoria, publicada en Florencia en 1485, Della statua y Della pittura. Donde una vez más son protagonistas las matemáticas, esta vez con cálculos exactos de números y con un objetivo, acercar las artes plásticas a las consonancias musicales.

En el De re aedificatoria, libro IX, cap. V, Alberti explica cómo, los intervalos musicales de octava, quinta y cuarta son agradables al oído, se corresponden a la división de la cuerda en 2,3 y 4 (1/2, 2/3 y 3/4), proporciones conocidas en la época como: diapasón, diapente y diatesarón. Estas proporciones servirán en la arquitectura, en la pintura y en la escultura, como una relación armónica que se instaurará entre las formas que, partiendo de un cuadrado, se diversificarán en rectángulos que tienen por lado $1 / 2,2 / 3$ o $3 / 4$, relaciones que se utilizarán para proporcionar las construcciones no solo en planta sino también en fachada. Estas proporciones se pueden utilizar duplicadas y combinadas. Se definen así los números musicales, como los siguientes: uno, dos, tres, cuatro.

En el siglo XV, en muchos edificios religiosos, existen relaciones entre el ancho de la nave y la altura de ellas o relaciones entre las medidas de la planta. Se puede encontrar, en diferentes libros, la conexión que hay entre el motete Nuper Rosarum Flores (figura 3), que el maestro flamenco Guillaume Dufay compuso para la consagración de la catedral de Florencia en 1436, y la planta de Santa María del Fiore; en estos textos se subraya que, en la composición vocal se utilizaron las relaciones que se mencionan en las medidas de la planta, en específico el largo de la nave, el ancho del transepto, el ancho del ábside y la altura de la catedral. También la cantidad de compases utilizados por Dufay, corresponden al módulo, en brazos, de la catedral. Es interesante ver en estos libros la partitura musical, el esquema formal del motete y su relación con la planta de la catedral.
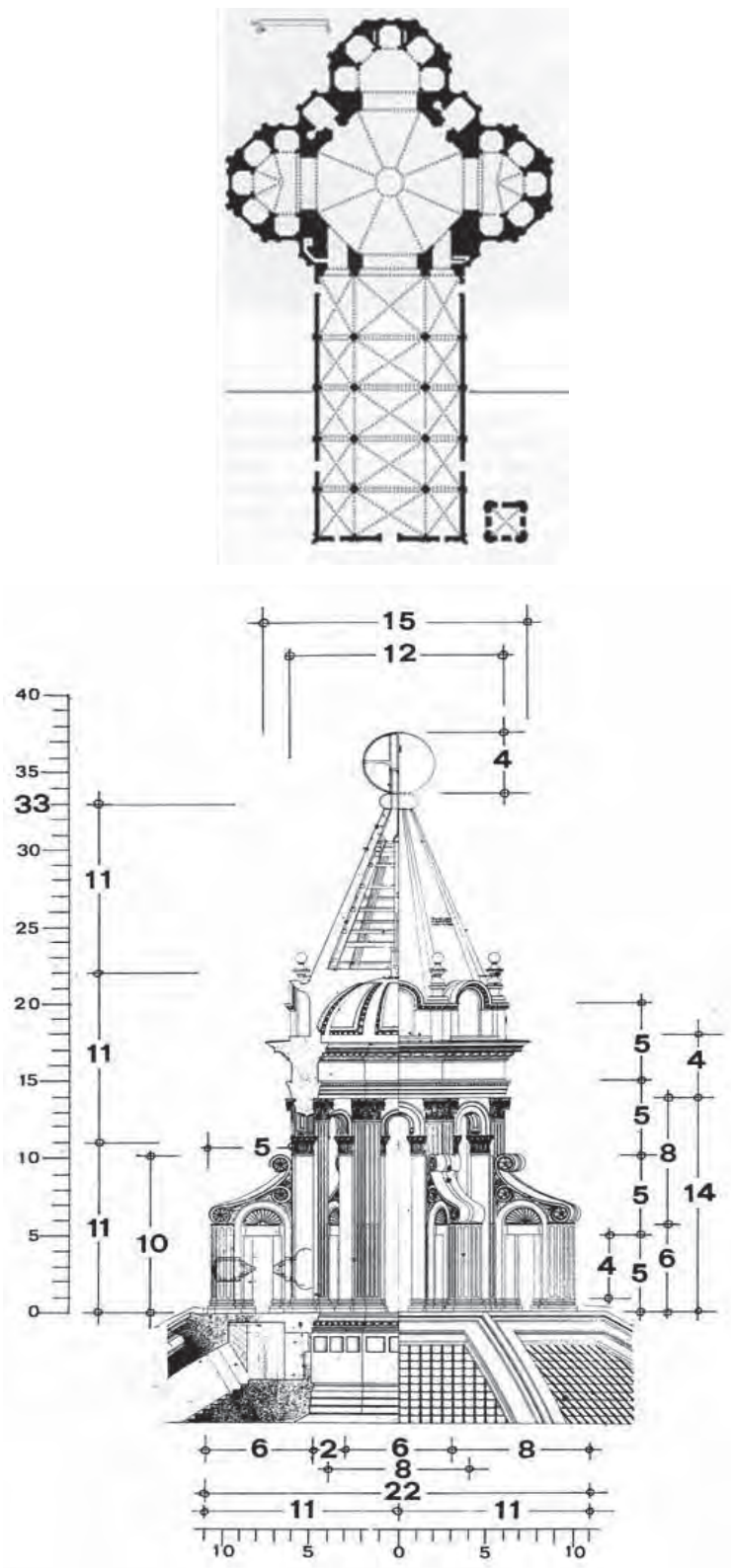

Figura 3. Si se observa la estructura de la catedral, se notará la repetición de algunos números, la modulación está dada por un bloque que equivale a 28 brazos, la nave está compuesta por 6 módulos, el transepto por dos módulos por un total de cuatro, la zona absidal por dos módulos, mientras tres módulos separan el techo de la cúpula a la cota del pavimento. Regresa el esquema 6:4:2:3 como el de los motetos (figura 4) 


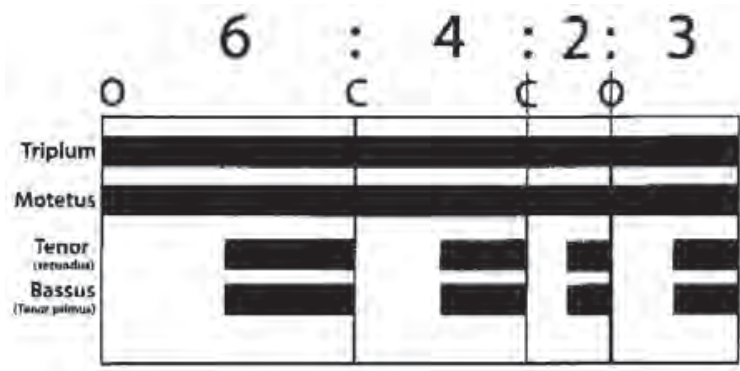

Figura 4. En las letras pequeñas: Triplum - Motetus Tenor (secundus) - Bassus (primus)

Palladio, como escribe en el primero de Los cuatro libros de la Arquitectura (figura 6), él trata las proporciones de las habitaciones y elenca las mejores siete que se pueden utilizar en las plantas, en orden: la circular, la cuadrada y la rectangular $\sqrt{ } 2 / 1$ (diagonal del cuadrado), 4/3 (un cuadrado y un tercio), 3/2 (un cuadrado y medio), $5 / 3$ (un cuadrado y dos tercios) y $2 / 1$ (dos cuadrados) concluye diciendo que evita las relaciones largo-ancho mayores a 2/1. En otro capítulo, define las proporciones para las alturas de las habitaciones.

Rudolf Wittkower, en Principi architettonici nell'età dell'Umanesimo, ${ }^{2}$ escribe que las relaciones preferidas utilizadas por Palladio en su tratado se podían interpretar como relaciones musicales, ya afirmadas en el siglo XVI. En una escala mayor cualquiera (no temperada), las correspondientes relaciones entre las alturas de los sonidos son las siguientes (para el ejemplo usa una escala de Do Mayor por la ausencia de alteraciones, pero la relación queda la misma para cualquier escala mayor no temperada):

Do/Do $=1 / 1=$ Primera Justa o Unísono

$\mathrm{Do} / \mathrm{Re}=9 / 8=$ Segunda Mayor

$\mathrm{Do} / \mathrm{Mi}=5 / 4=$ Tercera Mayor

$\mathrm{Do} / \mathrm{Fa}=4 / 3=$ Cuarta Justa

Do/Sol $=3 / 2=$ Quinta Justa

Do/La $=5 / 3=$ Sexta Mayor

$\mathrm{Do} / \mathrm{Si}=15 / 8$ = Séptima Mayor

Do/Do $=2 / 1=$ Octava Justa

Palladio utilizó en los proyectos de sus villas y edificios estas correspondencias, muchas veces no coincide la medida del dibujo originario de la planta con la medida real de la edificación.
La manifestación de estas relaciones es clara, por ejemplo, en la Villa Capra, conocida como La Rotonda (figura 5) (Valmarana, 1566, Vicenza) donde se hace uso del unísono 58/58, $30 / 30$ es decir $1 / 1$. Otras relaciones utilizadas en las habitaciones son 26/15, 15/11 e 30/12, de los estudios de Wittkower.

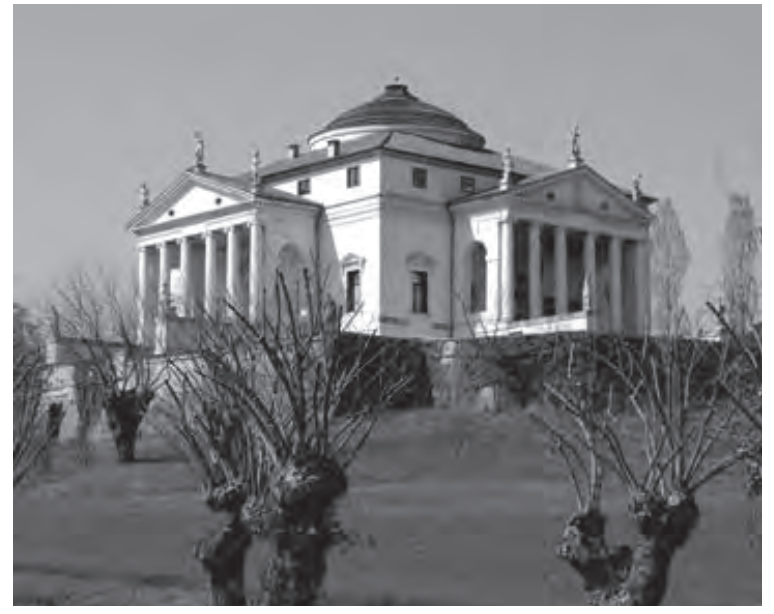

Figura 5. Andrea Palladio, Villa Capra llamada "La Rotonda", Vicenza

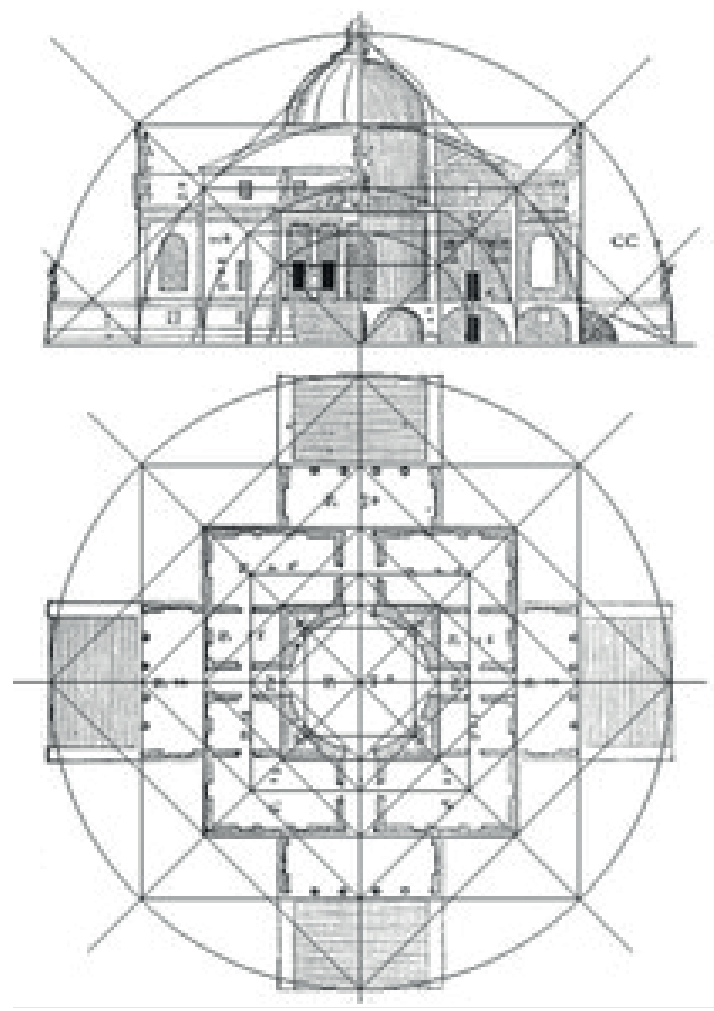

Figura 6. Planta obtenida del proyecto publicado en "I quattro libri dell'Architettura" del mismo Palladio y descomposición en círculos y cuadrados.

2 Traducción al español, Wittkower, Rudolph: Los fundamentos arquitectónicos en la edad del humanismo. Alianza Editorial, Madrid, 1995. N.E. 
Para citar alguna correspondencia de las habitaciones con las alturas de los sonidos, tómese el cuadrado del atrio central (30 x 30 brazos vicentinos), entre otros, que equivale a una relación de 1/1 o sea de unísono. Como es unísono también el espacio central que en planta es un círculo, es decir el rayo igual a 1/1. En realidad, toda la construcción se basa en la descomposición de cuadrados perfectos, como describe el mismo Palladio.

Es de este período el nacimiento del melodrama, en Florencia, donde se representó Euridice de Caccini y Peri -aunque aún no se puede hablar de verdadero melodrama hasta el S.XVIII-. Durante el Renacimiento faltando un lugar específico, las representaciones teatrales se efectuaban generalmente al aire libre, en los patios de los grandes palacios nobiliarios. Se considera el primer teatro estable al Teatro Olímpico de Vicenza de Palladio, el único que conserva aún hoy las escenografías originales. Durante los siglos XVII y XVIII nacen los teatros administrados por particulares, no más dentro de un edificio de corte, para convertirse en un lugar donde se entra pagando un billete, esta novedad abre la fruición a un público más amplio, muchas veces popular.

A partir de este momento el melodrama obligará la arquitectura teatral a una evolución que la llevará a los grandes teatros, símbolos del siglo XX, la arquitectura al servicio de la música.

\section{El escenario contemporáneo}

Se considera el final del siglo XIX y el comienzo del siglo XX como un momento de importantes cambios a nivel mundial, un momento de crisis que comporta la conclusión de la idea de perfección y de armonía. En campo artístico se descomponen, deconstruyen las precedentes representaciones figurativas. El siglo $\mathrm{XX}$ se presenta como un laboratorio del que parten muchas teorías correspondientes a las transformaciones dinámicas que se viven en ese momento. Una frase de Marinetti hace entender la situación "el dominio ilimitado de la intuición, el producto de la actividad incondicional imaginativa, son, entre las muchas desgracias de las que somos herederos, debemos reconocer que se nos ha dejado con la mayor libertad de espíritu”. (Marinetti, 1912).

También en música, después de la crisis del sistema tonal se da lugar a una frenética búsqueda de nuevos códigos lingüísticos sobre los que se basan las composiciones musicales; se regresa al uso de los modos, se adoptan nuevas escalas como la de tonos enteros, adoptada por Claude Debussy, como el cromatismo atonal y posteriormente dodecafónico, que tiende a alterar la dualidad entre consonancia y disonancia. Será Arnold Shönberg quien, junto a sus alumnos, delineará un nuevo sistema, conocido como dodecafonía.

Claude Debussy, el máximo exponente del Impresionismo musical, mostró su gran aversión a la armonía académica o escolar, él subraya una gran sensibilidad hacia los planos sonoros, dando gran espacio al timbre. Lo que hizo Debussy fue crear una nueva escala musical, la escala diatónica mayor, compuesta por 6 tonos enteros (sin semitonos), se trata del nacimiento de la escala esatonal. La escala esatonal de Do sería: Do, Re, Mi, Solb, Lab, Sib, Do2. Estos estudios llevarán indirectamente a la disolución de la tonalidad a través de la teoría dodecafónica de Arnold Shönberg (los doce sonidos de la escala cromática).

Nuevamente, las matemáticas son importantes en la música, Debussy hará uso en varias de sus obras de la proporción áurea - La mer matemáticas y ciencia tienen un rol importante en el aspecto metodológico compositivo. Shönberg libera sus obras del aspecto tonal por un sistema nuevo: atonal.

Xenakis utilizó este mismo concepto liberando la arquitectura de la forma arquitectónica conocida; el Pabellón de la Philips (Bruselas) es un gran ejemplo de esta colaboración.

No voy a tratar de las varias respuestas que la arquitectura ha dado en el siglo XX como oposición a la arquitectura del siglo XIX, sino de la complicidad compositiva que se crea entre arquitectura y música.

La música serial, que nace de la dodecafonía a la que añade una serie de variables como: 
ritmo, forma, timbre, altura y duración llega a crear una composición totalmente determinada en todos sus aspectos. Para poder visualizar este concepto, imaginemos una tabulación matricial con datos que se cruzan, los que tenemos en la barra vertical de la izquierda con los que tenemos en una barra perpendicular horizontal.

Es en el Pabellón de la Philips, de la Exposición Universal de Bruxelles de 1958, que Iannis Xenakis (1922 -2001) -músico, arquitecto, ingeniero y matemático- aplica las matemáticas de manera innovadora sea para la música que para la proyectación arquitectónica y aprovecha sus estudios de ingeniería para hacer cálculos y utilizar materiales adecuados. Su primera obra para orquesta fue Metastasis (1953-54), él estaba estudiando las alturas de dos acordes de doce sonidos cuando los une con una línea y se da cuenta de que podría ser un glissando. Glissando quiere decir subir o bajar de manera constante y progresiva la altura de un sonido, de esta manera no se percibe el pasaje entre las notas. Este mismo concepto lo utiliza en la proyectación arquitectónica del Pabellón. En 1948 Xenakis entra a trabajar en el estudio de Le Corbusier y colabora con él por 12 años.

En 1956, el director artístico de la Philips le pide a Le Corbusier que realice el proyecto del pabellón para la Exposición Internacional de Bruselas, donde se expondrá el campionario de productos electrónicos, en particular aquellos para el sonido y la luz, se le da máxima libertad para la creación de las fachadas, Le Corbusier respondió que no habría hecho un pabellón sino que habría creado un poema electrónico, donde el color, la imagen, el ritmo, el sonido y la arquitectura se habrían fundido de tal manera que el público habría quedado subyugado de todo lo que la Philips expusiera. Pidió también que pudieran participar en el proyecto dos grandes artistas a la vanguardia como él, Edgar Varese para la música proyectada al interior del Pabellón y Iannis Xenakis para el design y aspectos técnicos (figura 7).

En el atelier de Le Corbusier, Xenaquis fue encargado del desarrollo arquitectónico, cuenta este último "Me di cuenta de que, para determinar su forma, era necesario considerar muchos factores, pero mi investigación musical sobre los sonidos continuamente variables como una función del tiempo, me dirigieron hacia estructuras geométricas basadas en líneas rectas o en superficies generadas por curvas planas, es decir, los paraboloides hiperbólicos y los conoides" (figuras 7, 8 y 9). Fue la creación de una verdadera instalación artística, la primera arquitectura multimedia, capaz de despertar el sentido de una experiencia auditiva y visiva completa. El pabellón entonces, fue construido siguiendo una rigurosa y compleja elaboración geométrica de cáscaras ligeras, autoportantes, encurvadas hechas con placas pretensadas en cemento armado. El poema electrónico se representó en su interior, un espectáculo proyectado como una obra para orquesta, en la que los instrumentos virtuales eran las luces, los altavoces, las imágenes proyectadas sobre las superficies curvas, las sombras y las expresiones de los espectadores, en una identificación sustancial del espacio con el sonido. Dependiendo de como el espectador se moviera dentro del edificio, de hecho, cambiaría el conjunto de sus percepciones, tanto en lo visual como en el campo acústico, las paredes mismas, reflejando luces y notas, interactuarían con las personas estimulando diferentes sensaciones. Una síntesis de esta envergadura era demasiado vanguardista respecto a la contemporaneidad. Aunque tuvo más de dos millones de espectadores, fue destruido a los pocos meses de su inauguración, una grave pérdida para el mundo de la cultura.

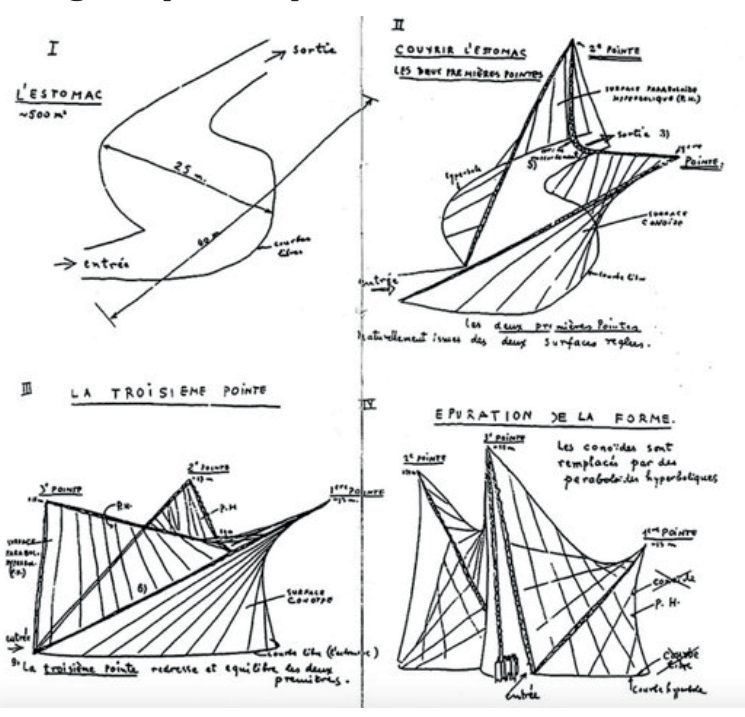

Figura 7. Bosquejos de Xenakis para el proyecto del pabellón, define la planta con la forma de un estómago. Elimina las paredes paralelas que producen reflexiones múltiples del sonido, las superficies curvas con rayo de curvatura variable son excelentes para obtener una reverberación adecuada. 


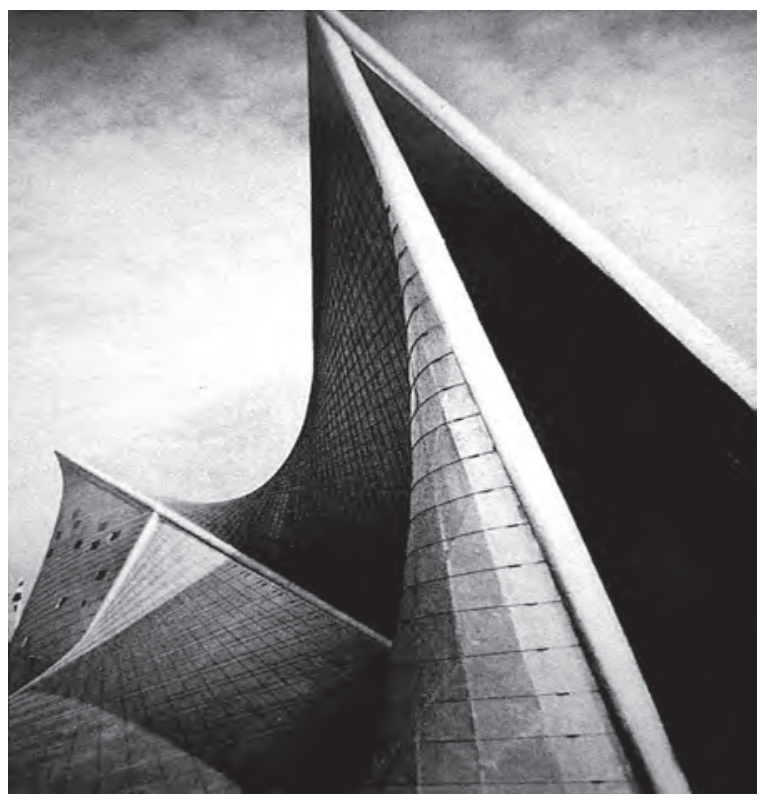

Figura 8. Se ve cómo los conoides han sido transformados en paraboloides hiperbólicos para dar una mayor facilidad a la construcción.

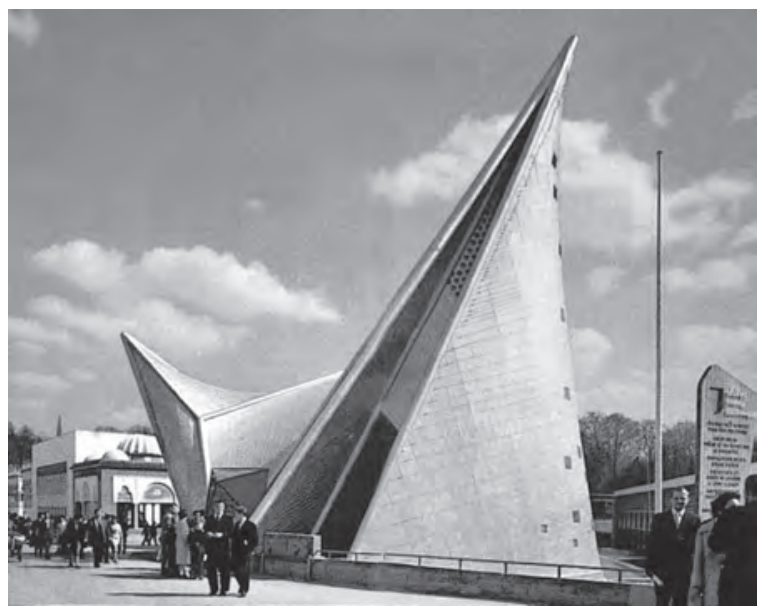

Figura 9. El Pabellón Philips terminado en 1958, Bruxelles.

Otro proyecto importante de Le Corbusier, en el que la música tiene un rol fundamental, aunque un poco escondido, es la capilla NotreDame-du-Haut de Ronchamp (1950-1955) (figuras 14 y 15). Aquí el arquitecto es capaz de recolectar y transmitir luz y sonido, como afirma Ruggero Pierantoni cuando investiga la "hermandad" de las sensaciones auditivas y visuales, es realmente capaz de construir "un puente entre dos provincias antiguas" del espíritu [el de las formas acústicas y el de las formas de luz]... Le Corbusier se refiere a esta construcción y dice que tiene una imagen de fuerte valor simbólico, que destruye "principios racionalistas, gramática y sintaxis". Sigue describiendo a Le Corbusier, el primer paso en esta intervención acústica fue encontrar una forma para transcodificar la forma visual y el efecto audible. Al igual que en la música, la arquitectura que aspira a una conexión con el sonido debe basarse en relaciones matemáticas, preceptoras de perfección y armonía.

Así como para el proyecto del Poema electrónico se parte de un estómago, aquí se parte de una campana (figuras 10 y 11) para alojar los sonidos y la armonía de la creación, y la oreja, órgano para escuchar el ambiente perceptible, puede ser fascinado por la flexibilidad, ligereza y precisión del fenómeno acústico.

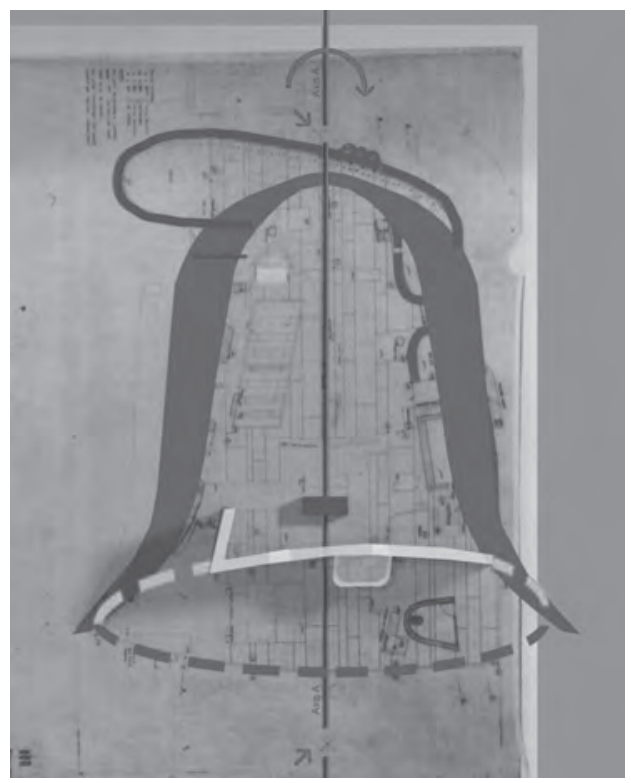

Figura 10. Planta de la capilla y sugestión de la campana

Pero no quiero subrayar la atmósfera que emana la arquitectura, sino dedicar unas líneas a las fachadas. Como dicen las actas de un Congreso dedicado a conmemorar a Le Corbusier: International Congress, LC 2015 Le Corbusier, 50 years later, realizado por la Universidad de Valencia en el 2015; las aberturas en las fachadas son la expresión de un estudio a pleno título de una composición musical. Ir a través de las fachadas, estas aberturas que recuerdan al rodillo perforado usado en las pianolas a finales del siglo XIX y principios del siglo XX, mientras que la elevación, en su conjunto, se asemeja a una hoja de música, notas escritas en la superficie de las paredes. Este mapa musical de las fachadas tiene un paralelo en 
los experimentos realizados en los años 50 por los compositores Edgard Varrse y Karlheinz Stockhausen (figura 13)... En este nuevo sistema de expresión, la notación musical había abandonado las marcas habituales codificadas en el pentagrama a favor de un nuevo modo de representación.

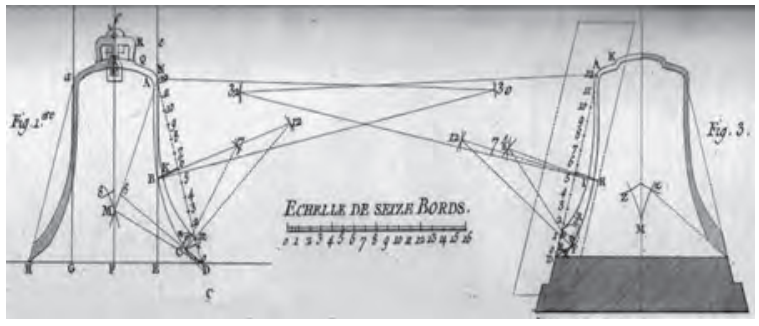

Figura 11. Representación de una campana en la enciclopedia de Denis Diderot y Jean-Baptiste Le Rond d'Alembert.

En realidad, este trabajo comprendía también la escritura de una partitura, partiendo de las aberturas y asignando líneas melódicas a diferentes instrumentos; posteriormente, dispusieron en una cuadrícula con el tiempo en las abscisas (x) y la altura de las notas musicales en el eje de ordenadas (y); esta información luego la insertaron en un software de música moderna, logrando un resultado en línea con las tendencias musicales actuales desde el momento que la capilla se diseñó en los años 50. Concluyen afirmando que, aunque las investigaciones en esta área continúan, los hallazgos hasta ahora, permiten afirmar que, como fue el caso del pabellón de Philips, Notre-Dame-du-Haut es en sí misma la fuente del sonido (una campana); casi para atestiguar que la arquitectura y la música pueden coincidir en armonía -.

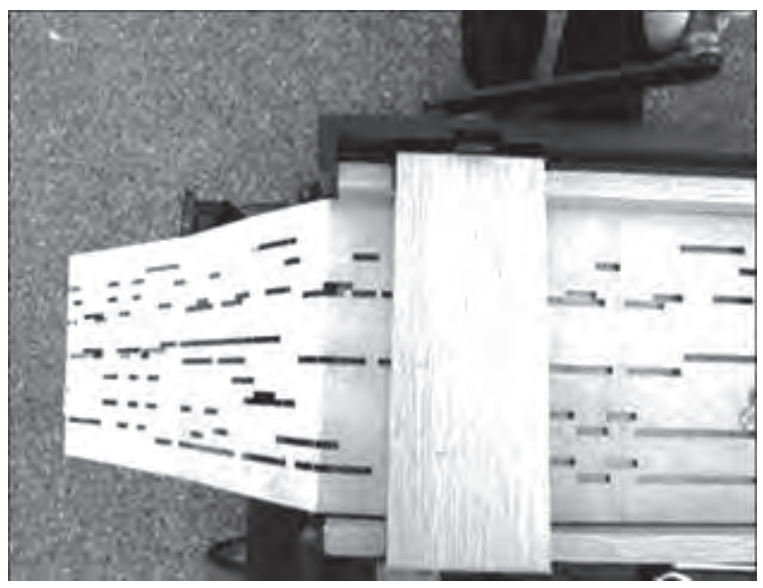

Figura 12. Disposición mecánica de un órgano

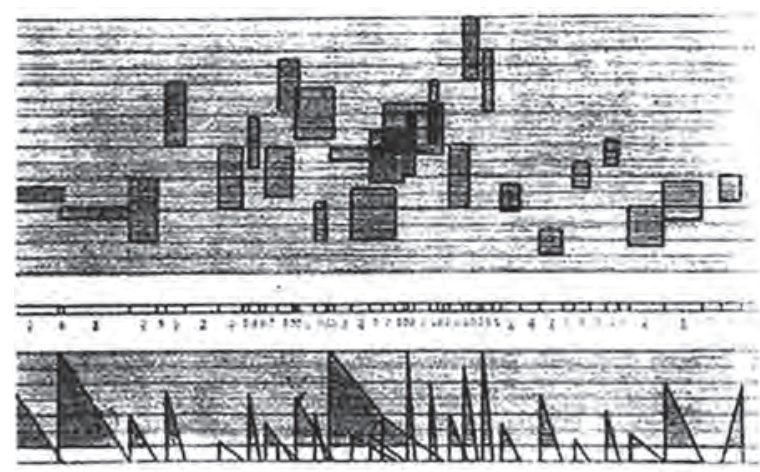

Figura 13. Karlheinz Stockhausen, Studie II, 1954

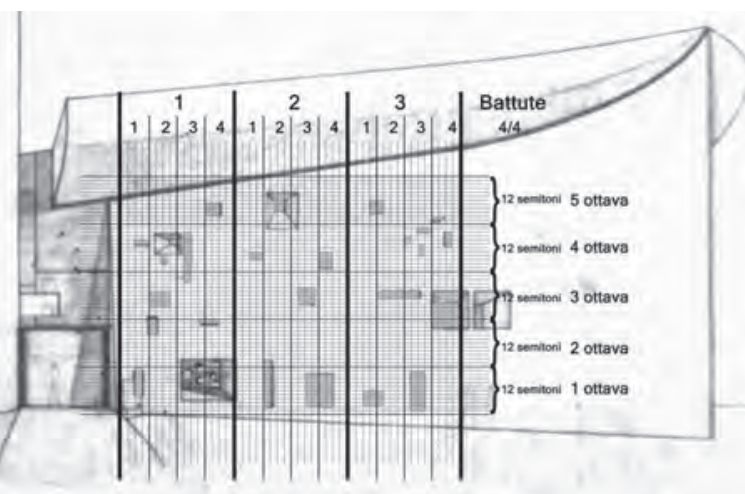

Figura 14. Fachada sur de la Capilla de Ronchamp

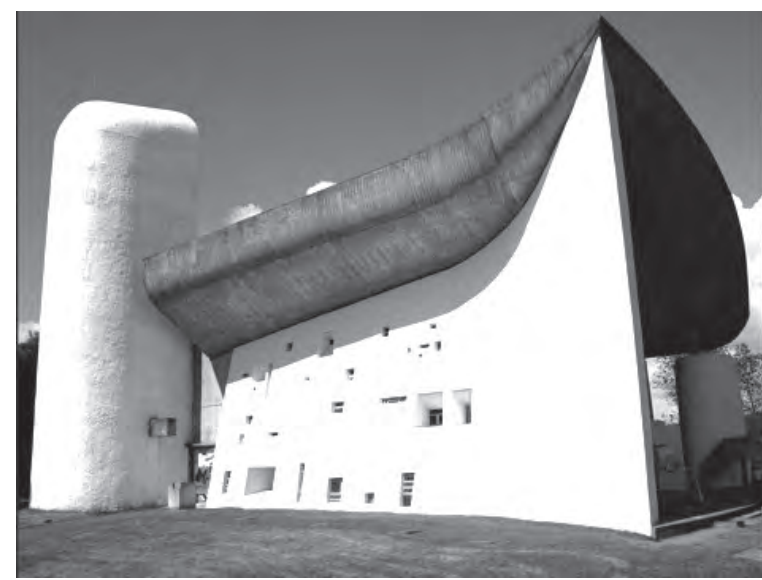

Figura 15. foto de la fachada sur de la Capilla de Ronchamp

En el siglo pasado, se desarrollaron muchos estilos musicales, Gershwin que logra mezclar la música culta europea con la música jazz; el blues, la música ligera, la comercial, el rap, el heavy metal y el pop, además de todas las fusiones entre música rock y músicas tradicionales de las diferentes civilizaciones de nuestro planeta.

Mientras la investigación arquitectónica después del International Style se desarrollará 
hacia corrientes como el Neorrealismo, el Postmodernismo, la arquitectura High Tech, el deconstructivismo o arquitectura blob, típica de Frank Ghery y Zaha Hadid.

Para terminar, citaré dos obras del arquitecto italiano Renzo Piano.

Cuando en 1971 Renzo Piano, junto a Richard Rogers, ganan el concurso para la realización del Centro George Pompidou de París, no estaba incluido el proyecto del IRCAM (Centro Internacional de Investigación Acústica Musical) (figura 16) se puede señalar este el inicio de Piano al mundo de la música, gracias a su colaboración con Pierre Boulez (presidente del IRCAM del 1977 al 1992) y Luciano Berio (director de la sección electro-acústica del 74 al 80); el proyecto nace como un lugar de encuentro para científicos y artistas que trabajan juntos en física y modificación de sonido, transmisión de señales, psicología cognitiva, musicología y creación musical.

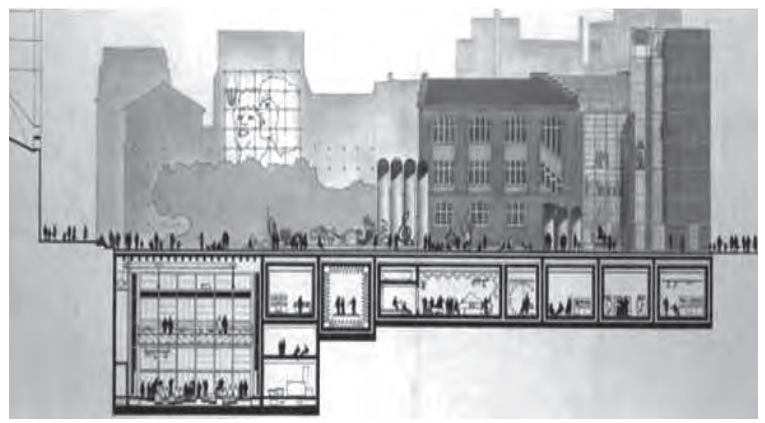

Figura 16. Sección transversal del IRCAM, París

Ya marcado por esta sensibilidad, es interesante su colaboración con el compositor Luigi Nono (1924-1990), personaje emblemático y representativo de la complicidad entre música y arquitectura, en la obra musical Prometeo (figura 18), con textos de Massimo Cacciari, Piano crea el espacio musical, poniendo al centro el edificio que, en este caso, es contenedor de música y caja de resonancia al mismo tiempo.

Para Luigi Nono, conocido como el arquitecto de los sonidos, es importante integrar el espacio arquitectónico en sus composiciones; en su obra Prometeo, tragedia del escuchar (figura 17), el tema importante es escuchar, vivir la música, entonar el espacio, en este caso, estimular emociones e inquietudes que la música dodecafónica lleva consigo (figura 19). El Arca es el resultado de Piano, (figuras 20 y 21) en 1984 estrenan la obra al interior de una iglesia desconsagrada, San Lorenzo en Venezia.

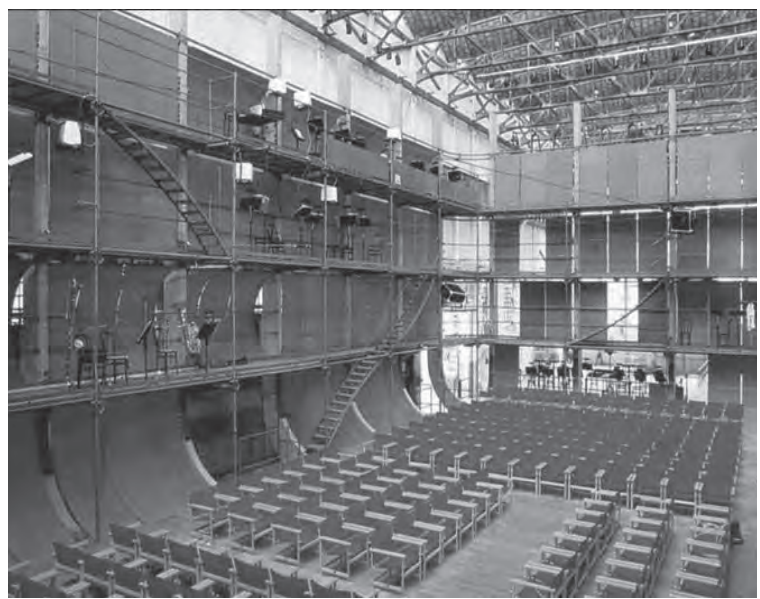

Figura 17. Luigi Nono. Prometeo

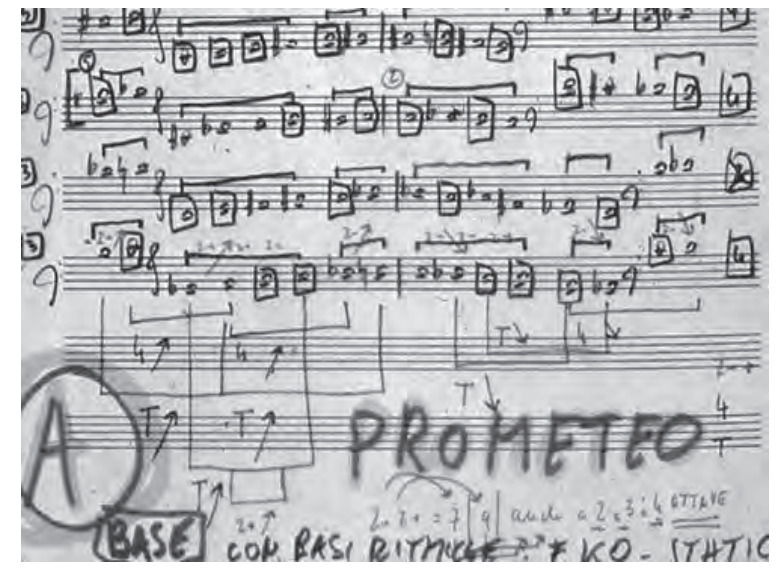

Figura 18. Una hoja de la partitura musical del Prometeo

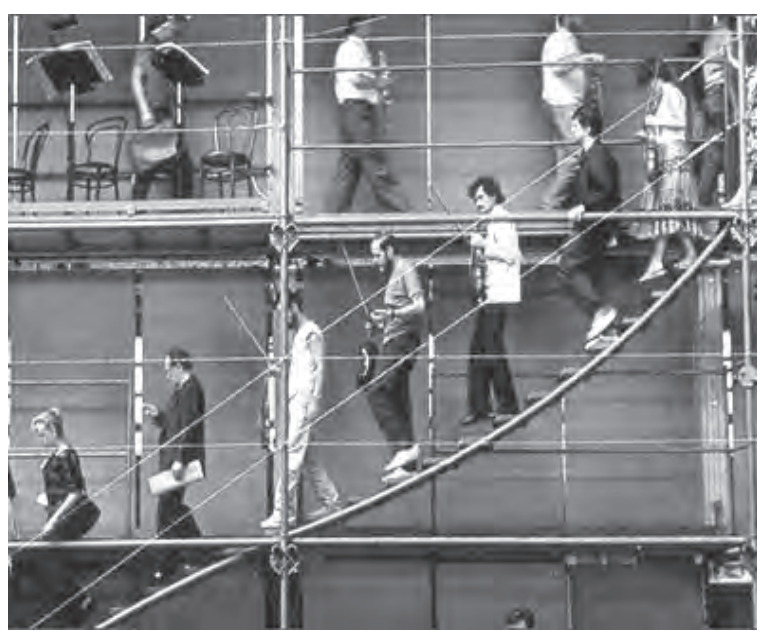

Figura 19. Los músicos están dispuestos en varios niveles, circundando al público, el objetivo es crear una interacción natural entre espacio y música. 


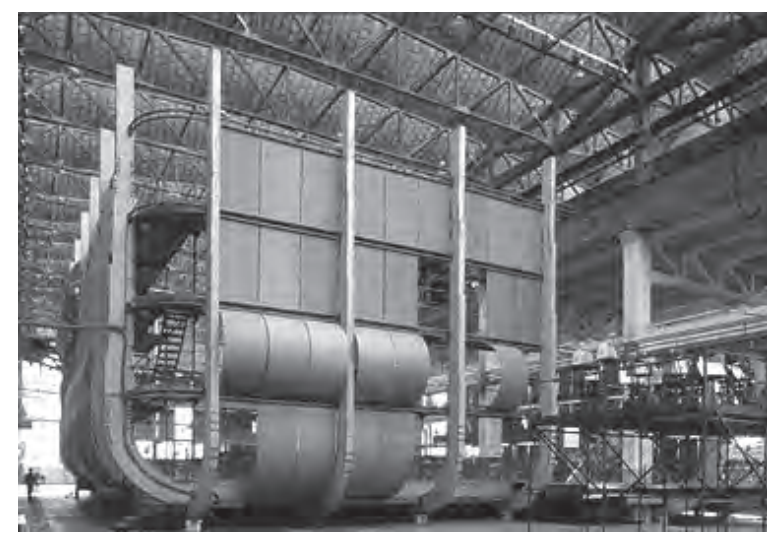

Figura 20. Renzo Piano. El Arca

Una particularidad de esta estructura es que es desmontable, modular y recomponible. Realizada en madera, por las propiedades acústicas del material. Muchas eran las características que se tenían que tener en consideración, como la dirección múltiple de la orquesta y coro, a través de pantallas o el movimiento de los coristas durante el espectáculo. En realidad, se piensa en un espacio arquitectónico como instrumento acústico. El resultado fue de lo más intrigante $y$ cautivador a nivel emotivo, como observaron los críticos.

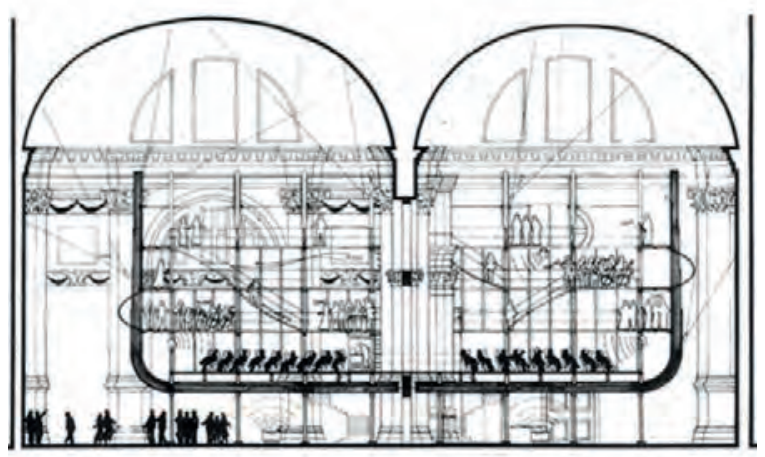

Figura 21. Sección del proyecto para el Prometeo Renzo Piano dice "Es el sonido que ordena, es la caja armónica la que debe poder vibrar con sus frecuencias y su energía”.
En el curso de su carrera, Piano ha tenido la oportunidad de construir a menudo para la música: del Instituto de Investigación Acústica Musical en París con Pierre Boulez y Luciano Berio, al Prometeo con Luigi Nono, a la sala de Lingotto en Turín (1983-1985), al Centro cultural "Jean-Marie Tjibaou" en Nouméa - Nueva Caledonia (1991-1998), al salón de Berlín en Potsdamer Platz (1992 2000), a la Sala Niccoln Paganini en Parma (1997-2001), al Auditorio Parque de la Música de Roma (1994-2002), al Auditorium del Banco Popular de Lodi (2000-2009), al Auditorium del Parque de L'Aquila (2009. 2012), entre otras obras.

\section{CONCLUSIONES}

Podríamos seguir citando relaciones entre música y arquitectura, ¿̇cuál de las dos influye en la otra? Creo que no es importante esta pregunta, es más importante ver cómo una inspira a la otra. ¿El espacio es para la música y el tiempo para la arquitectura? Me gusta pensar como arquitecto, que - la música se funde en el alma del mundo y del universo, atribuyéndole las resonancias en las esferas que, prescindiendo de la posibilidad de que sean perceptibles, son tangibles, símbolo de perfección absoluta - (Platón) y ella, la música, seguirá inspirando generaciones de arquitectos y viceversa. 


\section{REFERENCIAS}

Allorto, Riccardo, 2005. Nuova storia della musica, Ricordi. Milano.

Bouleau, Charles. 1996. La geometria segreta dei pittori. Mondadori Electa. Milano.

Brooks Allen, H. 1993. Le Corbusier 1887-1995, Electa. Milano.

Capanna, Alessandra. 2000. Le Corbusier. Padiglione Philips, Bruxelles, en Testo\&Immagine. 67 Turin.

Dall'Olio, Lorenzo. 1997. Arte e architettura. Nuove corrispondenze. Testo\&Immagine, 30. Turin.

Docci Mario 2012. Rapporti armonici in architettura, Musica \& Architettura, Nuova Cultura. La Sapienza.Roma.

Favaro Roberto.2010. Spazio sonoro. Musica e architettura tra analogie, riflessi, complicità, Saggi Marsilio. Padova.

Jenger Jean. 1997 Le Corbusier. L'architettura come armonia, Universale Electa/Gallimard, 1997 Milano.

Mila Massimo. 1963. Breve Storia della musica, Einaudi. Torino.

Milla Villena Carlos. 2011., Génesis de la Cultura andina. Amaru Wayra. Lima.

Marinelli Mauro. 2012 Consonanza e dissonanza. Strutture armoniche e ritmiche dello spazio: l'Acropoli di Atene, in Musica\&Architettura. Nuova Cultura. La Sapienza. Roma.

Palladio, Andrea. (1570) 1990. I quattro libri dell'architettura, reproducción editada por Hoepli. Milano

Vitruvio Polion, Marco. (s. I dC.) 1997 De Architectura, Libri decem. Libro III, cap.1, de Pierre Gross, Eunaudi editore. Torino.

Wittkower Rudolph. 2007. Principi architettonici nell'età dell'Umanesimo, Einaudi editore. Torino.

\section{ARTÍCULOS EN LÍNEA}

Ackerman J., Ricordi della nona triennale, De Divina Proportione.

https://elichenuove.files.wordpress.com/2012/11/la-divina-proporzione.pdf

Armónicos del sonido explicados por Leonard Bernstein

http:/www.musica-spirito.it/musica-scienza/armonici-del-suono-spiegati-da-bernstein/

Auditorium della Musica. fondazionerenzopiano.org

auditorium.com

iuav.it

it.wikipedia.org 
Bergson H., citado en Che rapporti ci sono tra musica e architettura? Claudio Ferrarini, 9/09/2011

https://claudioferrarini.wordpress.com/2011/09/09/che-rapporti-ci-sono-tra-musica-earchitettura/

El Pabellón de la Philips, Xenakis, Le Corbusier citado en:

https://philomenegattuso.wordpress.com/2005/11/26/post-30/

La musica nel pensiero pitagorico - Oilproject - WeSchool

https://library.weschool.com/lezione/la-musica-nel-pensiero-pitagorico-20523.html

a música en el Antiguo Egipto. Curso del C.A.Egiptología.

http://www.archeofriuli.it/wp-content/uploads/2016/11/musica\%20egizia.pdf

La sección áurea en música y el intervalo de sexta, citada en:

https://www.musica-spirito.it/musica-2/sezione-aurea-musica-intervallo-di-sesta/

Luigi Nono y Renzo Piano citados

https://eutopics.wordpress.com/2013/01/09/musica-come-spazio-abitabile-larca-di-renzo-pianoper-il-prometeo-di-luigi-nono-1983-1984/

Pitágoras citado en: Armonia di Musica e Architettura

https://www.musica-spirito.it/musica-2/armonia-di-musica-e-architettura/

Fecha de recepción: 30-03-18.

Fecha de aceptación: 09-05-18. 